\section{Linear Intercepts, Areas and Volumes}

In recent correspondence, Mr. P. A. P. Moran ${ }^{1}$ has suggested a simple method of finding the surface area of small objects from their average area of projection. Another simple method of finding the surface area and also the volume of small objects is based on the measurement of their average linear intercept (mean chord). Some years ago, while working on the determination of the grain-size of rocks, I derived two formulæ and discovered two theorems related to the average linear intercepts. The formulæ can be applied to the calculation of the average grain-size in granular aggregates in which the sizes and the shapes of grains are the same. The theorems, so far as I can see, have a purely academic interest, although their application to granular aggregates may be attempted.

Enlarging on the conclusions arrived at by M. W. Crofton $^{2}$ for convex figures, the two formulæ are derived as follows: (1) In a plane convex figure (polygon, circle) the average length of projection is equal to the perimeter divided by $\pi$, and the average linear intercept $(l)$ is equal to the area divided by the average length of projection :

$$
l=\frac{\pi \times \text { area }}{\text { perimeter }}
$$

(2) In a three-dimensional convex figure (polyhedron, sphere) the average area of projection is equal to the surface area divided by four, and the average linear intercept is equal to the volume divided by the average area of projection :

$$
l=\frac{4 \times \text { volume }}{\text { surface area }}
$$

As particular cases of these formulæ we have the following theorems :

1. The average linear intercept of a convex polygon circumscribed by a circle is equal to the average linear intercept of the circle.

2. The average linear intercept of a convex polyhedron circumscribed by a sphere is equal to the average linear intercept of the sphere.

In conclusion I would like to express my thanks to Dr. H. P. Mulholland and Dr. G. R. Goldsbrough, for their help in this matter.

King's College,

Neẉcastle upon Tyne.

${ }^{1}$ Nature, 154, 490 (1944).

'Crofton, M. W., Proc. London Math. Soc., 8, 304 (1877).

\section{Amplitude Effect in Cepheid Variables}

Is the interesting review ${ }^{1}$ of the pulsation theory in his George Darwin Lecture of 1943 before the Royal Astronomical Society, Prof. S. Rosseland has indicated the importance of unharmonic oscillations in explaining the Cepheid characteristics, and brought out the effect of the amplitude in lengthening the pulsational period, pointed out by Kluyver ${ }^{2}$ in 1937 , and imparting the characteristic skewness to the light and velocity curve.

In the special case, when the amplitude is of the order of a fourth of the radius, the star being composed of a homogeneous monatomic gas, on neglecting the overtone effect, and taking account of the self-coupling term for the fundamental up to the third order only, Prof. Rosseland mentions a lengthening of period in the ratio $1 \cdot 7: 1$, and a rise to the maximum four times faster than the decline to the minimum. While there is a considerable discrepancy to be bridged, these results should be taken as good indication of the amplitude effect.

For general values of the adiabatic index, it is, of course, imperative to take resort to approximations, even with a predominant self-coupling term; but for homogeneous monatomic gases, a complete solution for the fundamental can be obtained. One of the integrals of motion, since the time is not explicitly present in the Hamiltonian, is

$$
H=K+U+V=\text { const., }
$$

$K, U, V$ being the kinetic, thermal and potential energy of the star. Neglecting the overtone velocity term in $K$, and the inter-coupling terms in $U$ and $V$, the equation for the time coeiticient can be solved without approximation, and it is found that assuming $\eta$ to be the proportionate amplitude of pulsation, the time coelficient varies between the limits 1 and $(1+2 \eta)^{-1}$. For $\eta=1 / 3$, this gives 1 and -0.6 ; the corresponding values given by the third order approximation are 1 and $-0 \cdot 5$. This is close enough, but at the same time the value -0.6 indicates a further increase in the discrepancy with the observed values.

The pulsational period in terms of that for the harmonic period is $\frac{T_{\text {anharmonic }}}{T_{\text {harmonic }}}=\frac{(1+\eta)^{3}}{(1+2 \eta)^{3 / 2}}$,

while the speed of increase of the velocity curve to maximum over the decline to the minimum is given by the ratio

$$
\frac{\text { Time of decline }}{\text { Time of rise }}=\frac{\pi-\cos ^{-1}\left(\frac{\eta}{(1+\eta)}\right)+\frac{\eta \sqrt{1+2 \eta}}{(1+\eta)^{2}}}{\cos ^{-1}\left(\frac{\eta}{1+\eta}\right)-\frac{\eta \sqrt{1+2 \eta}}{(1+\eta)^{2}}}
$$

These give a steady lengthening of the period from the value for the harmonic oscillation to a value 1.56 times as great. The skewness of the velocity curve increases as $\eta$ varies from 0 to 1 ; during this time the speed ratio increases only from 1 to $4 \cdot 12$. For $\eta=\frac{1}{8}$ these ratios are 1.20 and 1.92 , while Prof. Rosseland's values are $1 \cdot 70$ and 4 approximately. The details of the calculations will be given elsewhere.

Thus the fitting to the observed values achieved for the rather large value $\eta=\frac{1}{3}$ even vanishes, and the discrepancy which Prof. Rosseland rightly points out appears to be considerably greater than he thinks. For values of $\eta$ of the order of the observed. values, the anharmonic oscillation curves would be almost indistinguishable from the harmonic case.

We therefore think that the amplitude effect is a contributory cause to the Cepheid characteristics, and probably not a dominant cause; and factors such as the decrease in the central condensation ${ }^{3,4}$ must be brought in for a complete explanation of the Cepheid phenomenon.

Mathematics Department,

SUNIL Kumar Roy.

${ }^{1}$ Rosseland, S., Mon. Not. Roy. Ast. Soc., 103, 233 (1943).

Kluyver, H. A., B.A.N., 7, 265 (1937).

${ }^{3}$ Miller, J. C. P., Mon. Not. Roy. Ast. Soc., 90, 59 (1929).

${ }^{4}$ Kopal, Z., Mon. Not. Roy. Ast. Soc., 89, 33 (1939). 